\title{
Response of Some Citrus Rootstock Seedlings to Stimulating Growth Applications
}

\author{
M.A. Ibrahim*, Abeer T. Mohsen"*, M.A. Abdel-Mohsen"*, \\ and Amany S. Mostafa* \\ *Citrus Research Department, Horticulture Research Institute, \\ Agricultural Research Centre and ${ }^{* *}$ Pomology Department, \\ Faculty of Agriculture, Cairo University, Cairo, Egypt.
}

\begin{abstract}
7 HE PRESENT study was conducted under screen-house in the nursery of Horticulture Res. Inst., Giza, Egypt on Sour orange (Citrus aurantium L.) and Volkamer lemon (Citrus volkameriana) rootstock seedlings during the growing seasons of 2011 and 2012. The study aimed to the assess the influence of using phosphorus, active dry yeast, algae(diatoms) and their combinations on growth and leaf mineral contents " N, P and K" of the studied rootstocks. The obtained results showed an enhancement effect on both rootstocks performance. Moreover, seedlings treated with either active dry yeast, algae or phosphorus and remarkably their combinations showed significant augmentations of growth parameters i.e., stem length, stem thickness, leaf area, dry weight of leaves and roots. Leaves nitrogen, phosphorus and potassium content increased significantly with such applications.
\end{abstract}

Therefore, it is recommended to use the combination between active dry yeast, algae and phosphorus to improve seedlings vegetative characteristics and shorten the time seedling needs to attain grafting size.

Keywords: Citrus rootstocks, Sour orange, Volkamer lemon, Growth characters, Phosphorus, Active dry yeast, Algae and Diatoms.

Citrus is one of the most important world fruit crops grown in many tropical and subtropical countries. Citrus occupies a prominent position in the fruit industry of the world, as well as in Egypt. It is the first popular fruit crop in Egypt and the most important fruit crop regarding both production and consumption because of its high nutritional value and the length of displayed markets(El-Kassas, 1984). Moreover, it occupies about 541,723 feddans (227,524 ha) which represent $33.97 \%$ of total fruit cultivated area in Egypt during 2013 according to the yearly Bull. (Agricultural Economic and statistics- Ministry of Agriculture and land Reclamation of Egypt). During the past decades the Egyptian citrus industry has continued to increase. The number of nurseries has also increased to meet the demand caused by replanting, tree loss to aging and virus and virus-like diseases and industry expansion to new land (Latif, 2004).

Rootstocks are of vital importance in the quality and quantity of production and survival of citrus plants. Sour orange (Citrus aurantium L.) is a universal rootstock for citrus and widely used in the Mediterranean region (Ibrahim, 2007). Sour orange rootstock is reported to be suitable for heavy moist soil, producing 
high yield of good fruit quality. The produced fruits characterized by smaller size, thin and smooth skin, high TSS and low acidity (Hemeda, 2014). Volkamer lemon (Citrus volkameriana) used as a rootstock for citrus, due to its tolerance to Tristeza and its acceptable resistance of a large scale of citrus diseases. It has significant enhancement effect on growth due to its suitability for the unfavorable environmental (climatic and soil) conditions (Ibrahim, 2007).

Phosphorus (P) is one of the most important elements for plant growth and metabolism. It plays a key role in many physiological processes such as energy metabolism, the synthesis of nucleic acids and membranes, photosynthesis, respiration, nitrogen fixation, enzyme regulation (Raghothama, 2005). Further, bio fertilizers as algae or yeast extract are recommended for increasing the growth parameters of many plants (Hegab et al., 2005 and Abdelaal et al., 2013).

In this concern, this study was carried out to determine the optimal combinations that lead to increase the root system growth and plant nutritional status which is reflected directly on the vegetative growth and consequently reduce the time needed in the nursery for seedling production.

\section{Material and Methods}

The present study was carried out during two consecutive seasons 2011 and 2012 in the nursery of Hort. Res. Inst., Giza, Egypt.

Stimulative growth substances used

Phosphorus

In the form of phosphoric acid $\left(85 \% \mathrm{P}_{2} \mathrm{O}_{5}\right)$

Active dry yeast

Concerning chemical analysis of yeast contents;(Nagodawithana, 1991).

\begin{tabular}{|lcccc|}
\hline Protein & Nucleic acids & Carbohydrates & Lipids & Minerals \\
\hline $47 \%$ & $8 \%$ & $33 \%$ & $4 \%$ & $8 \%$ \\
\hline
\end{tabular}

Approximate composition of vitamins (mg/g):

\begin{tabular}{|ll|ll|}
\hline Thiamine $\quad 6-100$ & Biotin & 1.3 \\
\hline Riboflavin $35-50$ & Cholin & 4000 \\
\hline Niacin $300-500$ & Folic acid & $5-13$ \\
\hline Pyridoxine $\mathrm{HCl} 28$ & Vit-B12 & 0.001 \\
\hline Pantothenate70 & & \\
\hline
\end{tabular}

Approximate composition of minerals (mg/g):

\begin{tabular}{|l|l|l|l|l|l|l|l|l|}
\hline $\mathbf{K}$ & $\mathbf{P}$ & $\mathbf{C u}$ & $\mathbf{N i}$ & $\mathbf{S n}$ & $\mathbf{C r}$ & $\mathbf{M g}$ & $\mathbf{S ~ 3}$ & $\mathbf{C a}$ \\
\hline 21.00 & 13.50 & 8.00 & 3.00 & 3.00 & 2.20 & 1.65 & 0.90 & 0.75 \\
\hline $\mathbf{M o}$ & $\mathbf{Z n}$ & $\mathbf{L i}$ & $\mathbf{N a}$ & $\mathbf{S e}$ & $\mathbf{V a}$ & $\mathbf{S i}$ & $\mathbf{F e}$ & $\mathbf{M n}$ \\
\hline 0.40 & 0.17 & 0.17 & 0.12 & 0.10 & 0.04 & 0.03 & 0.02 & 0.02 \\
\hline
\end{tabular}

Egypt. J. Hort. Vol. 41, No. 2 (2014) 


\section{Algae (diatoms)}

Concerning chemical analysis of diatoms contents; to be found that averaged contents of Zeatin $489.88 \mathrm{mg} / 100 \mathrm{~g}, \mathrm{SiO}_{2} 46.56 \%$, Amino acids such as glutamic $5097.88 \mathrm{ppm}$, tyrosine $1599.89 \mathrm{ppm}$, organic acids as fumaric $485839.73 \mathrm{ppm}$ and citric 114390.25ppm. (Water \& Environment Research Institute, 2010)

\section{Experiment layout}

Phosphoric acid ( $85 \%)$ as a source of phosphorus, diatoms as a source of algae and active dry yeast as a source of yeast were used in this investigation. A preliminary experiment was conducted to determine the range of the concentrations of algae and phosphorus to be investigated in this study. Uniform and healthy seedlings of the two rootstocks were divided into two groups for each rootstock. Each group was divided into 4 sub groups containing 6 seedlings (replicates). Algae were studied at four concentrations (1.2, 2.4, 3.6 and $4.0 \mathrm{~g}$ per seedling), the other rootstock group was treated with four phosphorus concentrations $(0.6,1.2,1.8$ and $2.4 \mathrm{~cm}$ per seedling).

According to the preliminary data, three concentrations for phosphorus (1.2, 1.8 and $2.4 \mathrm{~cm}$ phosphoric acid (85\%) per seedling) and one concentration for algae $(4 \mathrm{~g})$ were chosen. The yeast was used at $0.5 \mathrm{~g}$ yeast plus $0.5 \mathrm{~g}$ sugar added to $250 \mathrm{~cm}$ of water and left for 12 hours to ferment. Uniform seeds of Sour orange and Volkamer lemon were planted in January of both study seasons. Two months after planting, 480 uniform and healthy seedlings of each rootstock were chosen and transplanted individually in black polyethylene bags with dimensions $15 \times 35 \mathrm{~cm}$, filled with sand : peat moss (3:1) and kept under screen-house.

Sixteen treatments for each rootstock were supplemented as ground applications. Each treatment replicated three times, each with 10 seedlings. The seedlings were treated every 10 days until the end of the experiment (10 months for each season). Besides, seedlings of all treatments were fertilized with recommended nutrient dose every week till the end of growing season according to ministry assessment of agriculture recommended dose.

\section{Growth parameter}

The following morphological and chemical parameters were recorded at the end of the experiments in both seasons.

\section{Morphological parameter}

Stem length $(\mathrm{cm})$, stem thickness $(\mathrm{cm})$, leaf area $\left(\mathrm{cm}^{2}\right)$ as average and total, and dry weight of leaves and roots (g) after drying at $70^{\circ} \mathrm{C}$.

To measure average and total leaf area, twenty mature leaf sample of each replicate were taken at the middle portion of shoots and by using a cork borer twenty disks $(1 \mathrm{~cm}$ diameter) of each were taken. Dry weight of each disk was measured then average leaf area was calculated Bleasdale (1987).

Leaf area $=$ dry wt. of leaf $\mathrm{x}$ disk area $/$ dry wt. of disk 
Then total leaf area was calculated as follows:

Total leaf area $=$ number of leaves $\mathrm{x}$ leaf area

Chemical parameters

On both seasons, 20 mature leaves from the middle portion of shoots of each replicate were taken and washed several times with tap water followed by distilled water and then dried at $70^{\circ} \mathrm{C}$ for dry matter estimation. Dried samples were finely grounded with a porcelain mortar and pestle and stored in small light bags for determination of N, P and K (labanauskas, 1966).Content of nitrogen in leaves was determined by the modified micro- kjeldahel method as described by Vanshouwenburg and Walinga (1978), leaf phosphorus content was determined color metrically according to the method of Jackson (1958) and flame photometer method (Corning 410) used according to Piper (1950) to determined potassium content.

\section{Statistical analysis of data}

The periodical measurements were arranged in split analysis. The obtained data was subjected to analysis of variance (ANOVA) according to Snedecor and Cochran (1989) with 32 treatments, each treatment comprised of 3 replicates. Mstat-C program was used to calculate least significant difference (LSD) to compare between means of treatments according to Waller and Duncan (1969) at probability of 0.05 using MSTAT software package. The data was tabulated and represented by Excel program where appropriate.

\section{Results}

\section{Morphological parameters}

Stem features (length \& thickness)

The data presented in Table 1 and Fig 1, 2 revealed that, stem length and thickness of Sour orange and Volkamer rootstocks were increased in response to growth stimulants in the two seasons. Phosphorus $(\mathrm{P})$ fertilizer instigated a significant increase of stem height and thickness. Such parameters increased linearly by increasing $(\mathrm{P})$ doses in both rootstocks. Moreover, vegetative growth such as seedling stem length and thickness, were significantly increased by applying active dry yeast or algae.

Furthermore, both parameters were enhanced with the combination of the three stimulative substances application followed by bilateral combination then application with each substance alone. It could be noted also that, there was an overall trends for the rootstocks under study for the two seasons as they get less values when subjected to algae or algae\& yeast treatments comparing to their applications with phosphors in any of at concentrations. Moreover, such charts clarify a steady trend for the algae its treatment since it obtains the least values for the seedlings stem thickness once it applied individually comparing the other stimulants. However, they achieved better results with the combination between the algae and whichever the other substances.

Egypt. J. Hort. Vol. 41, No. 2 (2014) 
The utmost values were obtained with the seedlings received the highest dose of $(\mathrm{P})$ plus algae and yeast during the two seasons.

TABLE 1. Effect of some stimulants on stem length of two citrus rootstocks during 2011 and 2012 seasons.

\begin{tabular}{|c|c|c|c|c|c|c|c|}
\hline \multirow{4}{*}{$\begin{array}{l}\text { Stimulative } \\
\text { substances }\end{array}$} & \multicolumn{7}{|c|}{ Stem length $(\mathrm{cm})$} \\
\hline & \multicolumn{3}{|c|}{2011 season } & \multicolumn{4}{|c|}{2012 season } \\
\hline & $\begin{array}{c}\text { Sour } \\
\text { orange }\end{array}$ & \begin{tabular}{|c|}
$\begin{array}{c}\text { Volkamer } \\
\text { lemon }\end{array}$ \\
\end{tabular} & Means & $\begin{array}{c}\text { Sour } \\
\text { orange }\end{array}$ & $\begin{array}{c}\text { Volkamer } \\
\text { lemon }\end{array}$ & \multicolumn{2}{|c|}{ Means } \\
\hline & $20.8 \mathrm{~m}$ & $20.37 \quad \mathrm{~m}$ & $20.59 \mathrm{~K}$ & $16.5 \quad \mathrm{p}$ & $20.51 \quad$ o & 18.50 & $\mathrm{~J}$ \\
\hline $\mathrm{P}_{1}(1.2 \mathrm{~cm})$ & 27.00 & 30.00 & $28.50 \quad \mathrm{~J}$ & 26.00 & $29.00 \quad \mathrm{j}-1$ & 27.50 & I \\
\hline $\mathrm{P}_{2}(1.8 \mathrm{~cm})$ & 29.00 & 30.67 & $29.84 \quad \mathrm{I}$ & $27.33 \quad 1-n$ & 29.67 i-k & 28.50 & $\mathrm{HI}$ \\
\hline $\mathrm{P}_{3}(2.4 \mathrm{~cm})$ & 30.00 & 32.00 & $31.00 \mathrm{G}$ & 29.00 & $33.00 \quad \mathrm{c}-\mathrm{e}$ & 31.00 & $\mathrm{EF}$ \\
\hline Algae & 29.00 & 28.00 & $28.50 \quad \mathrm{~J}$ & $28.67 \mathrm{k}-\mathrm{m}$ & $27.00 \mathrm{mn}$ & 27.84 & I \\
\hline $\mathrm{P}_{1}+$ Algae & 30.00 & 30.67 & $31.34 \mathrm{H}$ & 29.33 & $31.00 \mathrm{f}-\mathrm{i}$ & 30.17 & FG \\
\hline $\mathrm{P}_{2}+$ Algae & 31.67 & 32.00 & $31.84 \mathrm{~F}$ & 31.00 & $33.00 \quad c-e$ & 32.00 & $\mathrm{DE}$ \\
\hline $\mathrm{P}_{3}+$ Algae & 32.00 & 33.00 & $32.50 \quad \mathrm{E}$ & $33.00 \quad$ c-e & $35.00 \mathrm{ab}$ & 34.00 & $\mathrm{BC}$ \\
\hline Yeast & 30.33 & 30.33 & $30.33 \mathrm{G}$ & 29.67 & 29.33 i-k & 29.50 & $\mathrm{GH}$ \\
\hline $\mathrm{P}_{1}+$ Yeast & 32.67 & $31.33 \mathrm{gh}$ & $32.00 \mathrm{~F}$ & 31.67 e-h & $32.00 \mathrm{~d}-\mathrm{g}$ & 31.84 & $\mathrm{DE}$ \\
\hline $\mathrm{P}_{2}+$ Yeast & 33.33 & 32.00 & $32.67 \quad \mathrm{E}$ & 32.67 & $33.00 \quad \mathrm{c}-\mathrm{e}$ & 32.84 & $\mathrm{CD}$ \\
\hline $\mathrm{P}_{3}+$ Yeast & 34.00 & 32.67 & $33.34 \quad \mathrm{D}$ & 33.67 & $34.00 \quad \mathrm{bc}$ & 33.84 & $\mathrm{D}$ \\
\hline Algae + Yeast & 32.00 & 32.00 & $32.00 \mathrm{~F}$ & 31.00 & 30.00 h-k & 30.50 & FG \\
\hline $\begin{array}{l}\mathrm{P}_{1}+\text { Algae }+ \\
\text { Yeast }\end{array}$ & 34.33 & 33.33 & $33.83 \mathrm{C}$ & 33.67 b-d & $35.00 \mathrm{ab}$ & 34.34 & $\mathrm{AB}$ \\
\hline $\mathrm{P}_{2}+$ Algae + Yeast & 35.00 & 34.33 & $34.67 \mathrm{~B}$ & 34.00 & $35.33 \mathrm{ab}$ & 34.67 & $\mathrm{AB}$ \\
\hline $\mathrm{P}_{3}+$ Algae + Yeast & 35.33 & 35.33 & $35.33 \mathrm{~A}$ & 35.00 & 36.00 & 35.50 & \\
\hline Means & 30.79 & 30.83 & & 29.61 & 31.00 & & \\
\hline
\end{tabular}

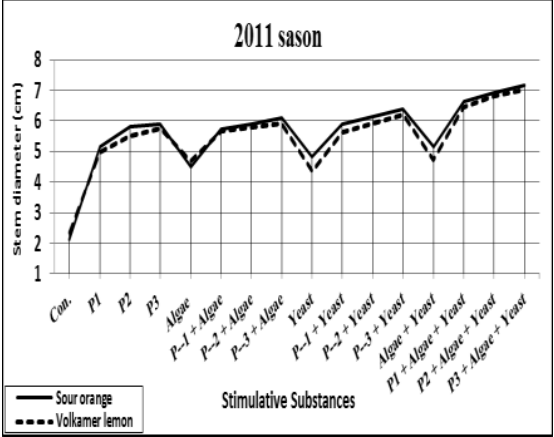

Fig. 1. Effect of some stimulants on stem thickness of two citrus rootstocks during 2011 seasons.

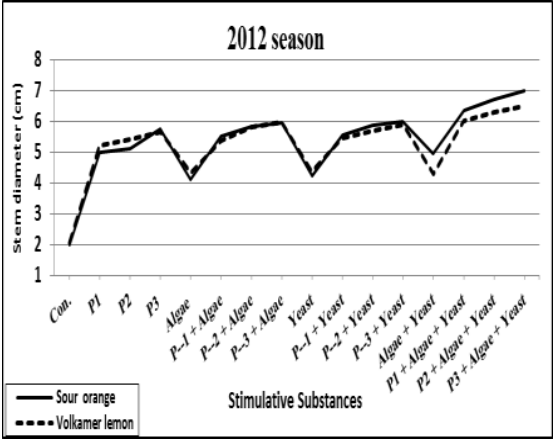

Fig. 2. Effect of some stimulants on stem thickness of two citrus rootstocks during 2012 seasons.

Egypt. J. Hort. Vol. 41, No. 2 (2014) 
Furthermore, it could be noticed that Volkamer lemon had the highest stem

length in both seasons even so it did not reach the significance level in the first one. Whereas, the Sour orange rootstock seedlings had significantly the largest stem thickness values in both seasons.

\section{Total and average leaf area}

Sour orange produced significantly higher average and total leaf area than Volkamer lemon in both seasons except for the average leaf area in the first season (Table 2). Each/all of the used stimulative substances enhanced significantly leaf parameters compared to control which got the lowest values. The presence of $(\mathrm{P})$ in nutrition solution increased leaf area. Moreover, the highest dose of $(\mathrm{P})$ enhanced rootstock average and total leaf area comparing to its other concentrations and to the other stimulants in single applications. Application of algae or yeast had positive effect on average and total leaf area but yeast applications were more effective in this respect. Moreover, these parameters were enlarged with three stimulative substances applications followed by bilateral combinations then with treating by either substance only. Significantly, highest average and total leaf area in both seasons was produced by rootstock seedlings received $\left(\mathrm{P}_{3}\right)$ plus algae and yeast in both seasons. Moreover, Growth criteria and average leaf area increased by diatoms (algae) treatment. Furthermore, active dry yeast proved to be an effective substance for achieving enhancement in leaf area.

It could be noted from Fig. $3 \& 4$ that, there were a significant decline in average and total leaf area expansion by adding algae or yeast, algae \& yeast comparing to their applications combined with phosphorus in both seasons. Otherwise, Yeast applications appeared to be more effective on average leaf area compared to phosphorus at medium and low doses applications or algae application. However, algae treatments generally had the lowest values of the average and total leaf area on Sour orange and Volkamer lemon rootstocks compared to the other stimulant applications in both seasons.

\section{Leaf dry weight}

The data presented in Table 3 showed that whichever the simulative substances were used, they enhanced significantly leaves dry weight of the citrus rootstocks under study compared to control during both seasons.

Phosphorus (P) fertilizer increased plant dry weight. Furthermore, it could be noted from the tabulated data that, as much as the $(\mathrm{P})$ concentrations get higher as much as the dry weight of leaves get more mass. Despite applying algae had improved plant dry weight, yeast applications had been more effective in this respect. Moreover, the high concentration of the phosphorus $\left(\mathrm{P}_{3}\right)$ surpassed each of the other stimulants either combined or uncombined.

Appling the three mingled stimulants applications enhanced dry weights of leaves more than treating with two of them followed by single substances applications (Fig. 5, 6). Accordingly, applying $\left(\mathrm{P}_{3}\right)$ combined with algae and yeast achieved the highest records for leaves dry weight for the studied rootstock

Egypt. J. Hort. Vol. 41, No. 2 (2014) 
seedlings in both seasons. On the other hand, the lowest value of dry weight of leaves was recorded with control of both rootstocks under study.

TABLE 2. Effect of some stimulants on total leaf area of two citrus rootstocks during 2011 and 2012 seasons.

\begin{tabular}{|c|c|c|c|c|c|c|}
\hline \multirow{4}{*}{$\begin{array}{l}\begin{array}{c}\text { Stimulative } \\
\text { substances }\end{array} \\
\text { Control } \\
\end{array}$} & \multicolumn{6}{|c|}{ Total leaf area $\left(\mathrm{cm}^{2}\right)$} \\
\hline & \multicolumn{3}{|c|}{2011 season } & \multicolumn{3}{|c|}{2012 season } \\
\hline & Sour orange & $\begin{array}{c}\text { Volkamer } \\
\text { lemon }\end{array}$ & Means & Sour orange & $\begin{array}{c}\text { Volkamer } \\
\text { lemon }\end{array}$ & \multirow{2}{*}{$\begin{array}{c}\text { Means } \\
88.15 \mathrm{~K}\end{array}$} \\
\hline & 109.96 & $105.19 \quad \mathrm{q}$ & 107.58 & 84.10 & 95.72 & \\
\hline $\mathrm{P}_{1}(1.2 \mathrm{~cm})$ & $163.75 \mathrm{mn}$ & $167.73 \mathrm{~lm}$ & 165.74 & 137.06 & 112.86 & $124.96 \mathrm{I}$ \\
\hline $\mathrm{P}_{2}(1.8 \mathrm{~cm})$ & 183.71 & 181.08 & 182.40 & $157.61 \quad \mathrm{jk}$ & 127.18 & $142.40 \mathrm{H}$ \\
\hline $\mathrm{P}_{3}(2.4 \mathrm{~cm})$ & 206.07 & 196.61 & 201.34 & 171.63 & 148.51 & $160.07 \mathrm{~F}$ \\
\hline Algae & 157.09 & 139.67 & 148.38 & 119.61 & 112.32 & $115.97 \mathrm{~J}$ \\
\hline$P_{1}+$ Algae & 197.39 & 185.70 & 191.55 & 155.41 & 127.09 & $141.25 \mathrm{H}$ \\
\hline $\mathrm{P}_{2}+$ Algae & 201.20 & 205.40 & $203.30 \mathrm{GH}$ & 166.95 hi & 146.00 & $156.48 \mathrm{~F}$ \\
\hline $\mathrm{P}_{3}+$ Algae & 212.96 & $217.76 \mathrm{gh}$ & 215.36 & 178.05 & 162.17 & $170.11 \mathrm{E}$ \\
\hline Yeast & 165.28 & 144.45 & 154.87 & 126.68 & 103.71 & $115.20 \mathrm{~J}$ \\
\hline$P_{1}+$ Yeast & 217.75 & 196.61 & 207.18 & 159.47 & 138.59 & $149.03 \mathrm{G}$ \\
\hline $\mathrm{P}_{2}+$ Yeast & 234.36 & 222.00 & 228.18 & 174.67 & 157.51 & $166.09 \mathrm{E}$ \\
\hline $\mathrm{P}_{3}+$ Yeast & 249.28 & 234.88 & 242.08 & 193.71 & $171.33 \mathrm{gh}$ & $182.52 \mathrm{D}$ \\
\hline Algae + Yeast & 173.50 & 158.97 no & 166.24 & 138.08 & 115.67 & $126.88 \mathrm{I}$ \\
\hline$P_{1}+$ Algae + Yeast & 259.78 & 249.31 & 254.55 & 202.51 & 184.81 & $193.66 \mathrm{C}$ \\
\hline $\mathrm{P}_{2}+$ Algae + Yeast & 281.06 & 265.00 & 273.03 & 214.41 & 202.25 & $208.33 \mathrm{~B}$ \\
\hline $\mathrm{P}_{3}+$ Algae + Yeast & 306.71 & 293.93 & 300.32 & 227.25 & 214.77 & $221.01 \mathrm{~A}$ \\
\hline Means & 202.57 & $193.06 \quad$ B & & 159.03 & 140.68 & \\
\hline
\end{tabular}

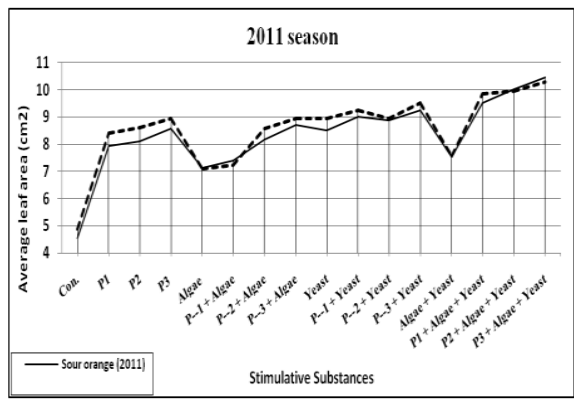

Fig. 3. Effect of some stimulants on average leaf area of two citrus rootstocks during 2011 seasons.

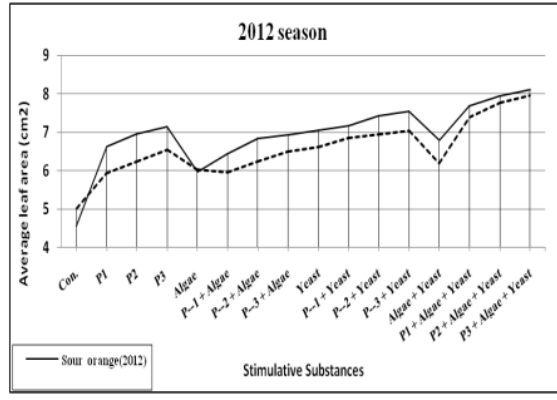

Fig. 4. Effect of some stimulants on average leaf area of two citrus rootstocks during 2012 seasons. 
TABLE 3. Effect of some stimulants on dry weight of leaves of two citrus rootstocks during 2011 and 2012 seasons.

\begin{tabular}{|c|c|c|c|c|c|c|c|c|c|c|c|c|}
\hline \multirow{4}{*}{$\begin{array}{l}\text { Stimulative } \\
\text { Substances } \\
\text { Control }\end{array}$} & \multicolumn{12}{|c|}{ Dry wt. of leaves (g) } \\
\hline & \multicolumn{6}{|c|}{ 2011season } & \multicolumn{6}{|c|}{2012 season } \\
\hline & \multicolumn{2}{|c|}{ Sour orange } & \multicolumn{2}{|c|}{$\begin{array}{c}\text { Volkamer } \\
\text { lemon }\end{array}$} & \multicolumn{2}{|c|}{ Means } & \multicolumn{2}{|c|}{ Sour orange } & \multicolumn{2}{|c|}{$\begin{array}{c}\text { Volkamer } \\
\text { lemon }\end{array}$} & \multicolumn{2}{|c|}{ Means } \\
\hline & 2.65 & $\mathrm{u}$ & 2.65 & $\mathrm{u}$ & 2.65 & $\mathrm{~N}$ & 2.73 & $\mathrm{~s}$ & 2.60 & & 2.67 & $\mathrm{~J}$ \\
\hline $\mathrm{P}_{1}(1.2 \mathrm{~cm})$ & 4.67 & o & 4.74 & no & 4.71 & $\mathrm{~J}$ & 4.00 & o & 3.84 & $\mathrm{p}$ & 3.90 & $\mathrm{H}$ \\
\hline $\mathrm{P}_{2}(1.8 \mathrm{~cm})$ & 4.84 & $\mathrm{mn}$ & 4.95 & $\operatorname{lm}$ & 4.90 & I & 4.54 & $\operatorname{lm}$ & 4.19 & $\mathrm{n}$ & 4.37 & G \\
\hline $\mathrm{P}_{3}(2.4 \mathrm{~cm})$ & 5.13 & $\mathrm{i}-\mathrm{k}$ & 5.25 & $\mathrm{i}$ & 5.19 & G & 4.81 & ij & 4.64 & $\mathrm{kl}$ & 4.73 & $\mathrm{~F}$ \\
\hline Algae & 3.46 & s & 3.25 & $\mathrm{t}$ & 3.36 & M & 3.55 & $q$ & 3.20 & $\mathrm{r}$ & 3.38 & I \\
\hline $\mathrm{P}_{1}+$ Algae & 4.73 & no & 5.12 & $\mathrm{jk}$ & 4.93 & I & 4.80 & ij & 3.95 & op & 4.38 & G \\
\hline $\mathrm{P}_{2}+$ Algae & 5.02 & $\mathrm{kl}$ & 5.43 & $\mathrm{~h}$ & 5.23 & G & 5.07 & gh & 4.50 & $\operatorname{lm}$ & 4.79 & $\mathrm{~F}$ \\
\hline $\mathrm{P}_{3}+$ Algae & 5.49 & gh & 5.78 & de & 5.64 & $\mathrm{E}$ & 5.25 & $\mathrm{f}$ & 4.92 & hi & 5.09 & $\mathrm{E}$ \\
\hline Yeast & 3.52 & rs & 3.62 & $\mathrm{qr}$ & 3.57 & $\mathrm{~L}$ & 3.63 & $q$ & 3.30 & $\mathrm{r}$ & 3.47 & I \\
\hline$P_{1}+$ Yeast & 4.87 & $\mathrm{~m}$ & 5.25 & $\mathrm{i}$ & 5.06 & $\mathrm{H}$ & 5.15 & fg & 4.40 & $\mathrm{~m}$ & 4.78 & $\mathrm{~F}$ \\
\hline $\mathrm{P}_{2}+$ Yeast & 5.23 & ij & 5.66 & ef & 5.45 & $\mathrm{~F}$ & 5.55 & $\mathrm{e}$ & 4.75 & $\mathrm{jk}$ & 5.15 & $\mathrm{E}$ \\
\hline $\mathrm{P}_{3}+$ Yeast & 5.62 & fg & 5.84 & $\mathrm{~d}$ & 5.73 & $\mathrm{D}$ & 5.85 & $\mathrm{~cd}$ & 5.23 & $\mathrm{f}$ & 5.54 & $\mathrm{D}$ \\
\hline Algae + Yeast & 3.94 & $\mathrm{p}$ & 3.72 & $\mathrm{q}$ & 3.83 & K & 3.90 & op & 3.74 & d & 3.82 & $\mathrm{~F}$ \\
\hline $\mathrm{P}_{1}+$ Algae + Yeast & 5.76 & de & 5.97 & $\mathrm{c}$ & 5.87 & $\mathrm{C}$ & 5.95 & $\mathrm{c}$ & 5.48 & $\mathrm{e}$ & 5.72 & C \\
\hline $\mathrm{P}_{2}+$ Algae + yeast & 5.88 & $\mathrm{~cd}$ & 6.29 & $\mathrm{~b}$ & 6.09 & B & 6.41 & $\mathrm{~b}$ & 5.75 & d & 6.08 & B \\
\hline $\mathrm{P}_{3}+$ Algae + yeast & 6.22 & $\mathrm{~b}$ & 6.66 & a & 6.44 & A & 6.60 & a & 5.89 & $\mathrm{~cd}$ & 6.25 & A \\
\hline Means & 4.71 & $\mathrm{~B}$ & 4.91 & A & & & 4.75 & A & 4.42 & B & & \\
\hline
\end{tabular}

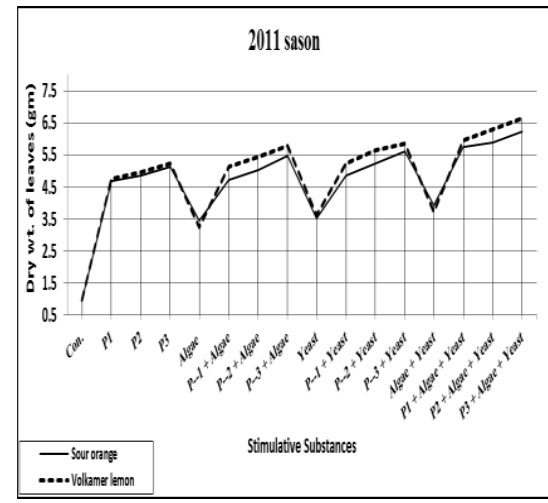

Fig. 5. Effect of some stimulants on leaf dry weight of two citrus rootstocks during 2011 seasons.

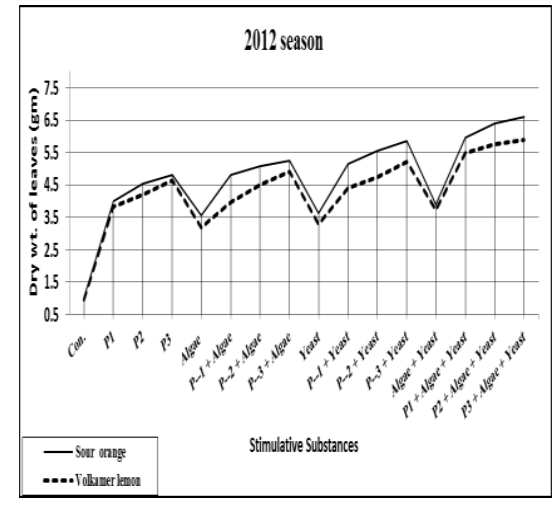

Fig. 6. Effect of some stimulants on leaf dry weight of two citrus rootstocks during 2012 seasons.

Egypt. J. Hort. Vol. 41, No. 2 (2014) 


\section{Root dry weight}

On the average, the Sour orange produced significant higher root dry weight measure up to Volkamer lemon in both seasons of the investigation (Table 4). (P) applications increased dry weight and length. Furthermore, root dry weight per seedling was linearly increased significantly with (P) doses increments.

Otherwise, the other two stimulants had a powerful effect on such parameter yet, yeast was more functional in this respect. While, phosphorus uppermost yeast records for both rootstock seedlings in both seasons even by applying the low or the medium $(\mathrm{P})$ concentrations.

Furthermore, dry weight of root was enhanced by the combination of the three stimulative substances applications followed by bilateral ones then treating with single substance. Such stimulants resulted in, the uppermost significant increments in root dry weight for the seedlings treated with the combinations of (P) at highest dose, algae and yeast. On the other hand, the lowest leaf area was recorded with both rootstocks under control treatment.

TABLE 4. Effect of some stimulants on root dry weight of two citrus rootstocks during 2011 and 2012 seasons.

\begin{tabular}{|c|c|c|c|c|c|c|c|c|c|c|c|c|}
\hline \multirow{4}{*}{$\begin{array}{l}\text { Stimulative } \\
\text { Substances }\end{array}$} & \multicolumn{12}{|c|}{ Total root dry weight(g) } \\
\hline & \multicolumn{6}{|c|}{ 2011season } & \multicolumn{6}{|c|}{2012 season } \\
\hline & \multicolumn{2}{|c|}{$\begin{array}{c}\text { Sour } \\
\text { orange }\end{array}$} & \multicolumn{2}{|c|}{$\begin{array}{c}\text { Volkamer } \\
\text { lemon }\end{array}$} & \multicolumn{2}{|c|}{ Means } & \multicolumn{2}{|c|}{$\begin{array}{c}\text { Sour } \\
\text { orange }\end{array}$} & \multicolumn{2}{|c|}{$\begin{array}{c}\text { Volkamer } \\
\text { lemon }\end{array}$} & \multicolumn{2}{|c|}{ Means } \\
\hline & 5.58 & $\mathrm{x}$ & 5.55 & $\mathrm{x}$ & 5.56 & $\mathrm{~N}$ & 5.73 & $\mathrm{q}$ & 5.64 & q & 5.68 & $\mathrm{~J}$ \\
\hline $\mathrm{P}_{1}(1.2 \mathrm{~cm})$ & 10.77 & $\mathrm{j}$ & 7.80 & q & 9.29 & $\mathrm{~J}$ & 7.73 & $\mathrm{~m}$ & 6.64 & o & 7.19 & $\mathrm{H}$ \\
\hline $\mathrm{P}_{2}(1.8 \mathrm{~cm})$ & 11.43 & $\mathrm{~h}$ & 8.79 & $\mathrm{p}$ & 10.11 & $\mathrm{H}$ & 8.11 & $\mathrm{jk}$ & 7.32 & $\mathrm{n}$ & 7.72 & G \\
\hline $\mathrm{P}_{3}(2.4 \mathrm{~cm})$ & 11.74 & $\mathrm{~g}$ & 9.43 & $\mathrm{n}$ & 10.59 & G & 9.00 & $\mathrm{~g}$ & 8.04 & $j-1$ & 8.52 & $\mathrm{~F}$ \\
\hline Algae & 7.27 & $\mathrm{t}$ & 6.03 & $\mathrm{w}$ & 6.65 & M & 7.75 & $\mathrm{~m}$ & 5.99 & $\mathrm{p}$ & 6.87 & I \\
\hline $\mathrm{P}_{1}+$ Algae & 11.45 & $\mathrm{~h}$ & 8.73 & $\mathrm{p}$ & 10.09 & $\mathrm{H}$ & 8.20 & ij & 7.26 & $\mathrm{n}$ & 7.73 & G \\
\hline $\mathrm{P}_{2}+$ Algae & 11.66 & $\mathrm{~g}$ & 9.43 & $\mathrm{n}$ & 10.55 & G & 9.00 & $\mathrm{~g}$ & 7.83 & $\mathrm{ml}$ & 8.42 & $\mathrm{~F}$ \\
\hline $\mathrm{P}_{3}+$ Algae & 12.12 & ef & 10.11 & 1 & 11.12 & $\mathrm{E}$ & 9.41 & $\mathrm{f}$ & 8.58 & $\mathrm{~h}$ & 9.00 & $\mathrm{E}$ \\
\hline Yeast & 7.49 & $\mathrm{~s}$ & 6.32 & $\mathrm{v}$ & 6.91 & $\mathrm{~L}$ & 7.69 & $\mathrm{~m}$ & 6.66 & o & 7.18 & $\mathrm{H}$ \\
\hline$P_{1}+$ Yeast & 9.47 & $\mathrm{n}$ & 9.28 & o & 9.38 & I & 9.49 & $\mathrm{f}$ & 7.72 & $\mathrm{~m}$ & 8.61 & $\mathrm{~F}$ \\
\hline $\mathrm{P}_{2}+$ Yeast & 12.05 & f & 9.99 & $\mathrm{~m}$ & 11.02 & $\mathrm{~F}$ & 9.57 & $\mathrm{f}$ & 8.40 & hi & 8.99 & $\mathrm{E}$ \\
\hline $\mathrm{P}_{3}+$ Yeast & 12.52 & d & 10.40 & $\mathrm{k}$ & 11.46 & $\mathrm{D}$ & 9.91 & $\mathrm{e}$ & 8.97 & g & 9.44 & $\mathrm{D}$ \\
\hline Algae + Yeast & 7.66 & $\mathrm{r}$ & 6.98 & $\mathrm{u}$ & 7.32 & K & 7.90 & $\mathrm{k}-\mathrm{m}$ & 7.20 & $\mathrm{n}$ & 7.55 & G \\
\hline $\mathrm{P}_{1}+$ Algae + Yeast & 12.77 & $\mathrm{c}$ & 11.19 & $\mathrm{i}$ & 11.98 & $\mathrm{C}$ & 10.36 & d & 9.94 & $\mathrm{e}$ & 10.15 & $\mathrm{C}$ \\
\hline $\begin{array}{l}\mathrm{P} 2+\text { Algae + } \\
\text { Yeast }\end{array}$ & 12.96 & a & 11.70 & $\mathrm{~g}$ & 12.33 & B & 11.28 & $\mathrm{~b}$ & 10.44 & d & 10.86 & B \\
\hline $\begin{array}{l}\text { P3 + Algae + } \\
\text { Yeast }\end{array}$ & 13.56 & $\mathrm{~b}$ & 12.23 & $\mathrm{e}$ & 12.90 & A & 11.85 & a & 10.81 & $\mathrm{c}$ & 11.33 & A \\
\hline Means & 10.42 & $A$ & 8.77 & $B$ & & & 8.70 & $A$ & 7.73 & $B$ & & \\
\hline
\end{tabular}


Chemical parameters

Leaf nitrogen $(N)$ content

The tabulated data (Table 5) revealed that there was no stable trend for the performance of the two rootstocks in relation to each other concerning leaf $\mathrm{N}$ content, as they switched over the domineering results for each season.

$\mathrm{N}$ content increased linearly- with lack of significant in some treatment differences -with increasing $(\mathrm{P})$ doses during both seasons, especially in the first one. Yeast had more positive effects on leaf nitrogen content compared to algae application. The mixed treatments increased significantly $\mathrm{N}$ leaf content measure up to the single ones. Furthermore, tri-combination treatments enhanced leaf $\mathrm{N}$ content and seedlings supplied $\left(\mathrm{P}_{3}\right)$ with algae plus yeast had the highest leaf $\mathrm{N}$ content. On other hand, the lowest leaf area was recorded with both rootstocks under control treatment.

TABLE 5. Effect of some stimulants on leaf nitrogen content of two citrus rootstocks during 2011 and 2012 seasons.

\begin{tabular}{|c|c|c|c|c|c|c|c|c|c|c|c|c|}
\hline \multirow{4}{*}{$\begin{array}{l}\text { Stimulative } \\
\text { Substances } \\
\text { Control }\end{array}$} & \multicolumn{12}{|c|}{ Nitrogen ( \%) } \\
\hline & \multicolumn{6}{|c|}{ 2011season } & \multicolumn{6}{|c|}{2012 season } \\
\hline & \multicolumn{2}{|c|}{$\begin{array}{c}\text { Sour } \\
\text { orange }\end{array}$} & \multicolumn{2}{|c|}{$\begin{array}{c}\text { Volkamer } \\
\text { lemon }\end{array}$} & \multicolumn{2}{|c|}{ Means } & \multicolumn{2}{|c|}{$\begin{array}{c}\text { Sour } \\
\text { orange }\end{array}$} & \multicolumn{2}{|c|}{$\begin{array}{c}\text { Volkamer } \\
\text { lemon }\end{array}$} & \multicolumn{2}{|c|}{ Means } \\
\hline & 1.34 & & 1.26 & $\mathrm{n}$ & 1.30 & $\mathrm{~J}$ & 1.31 & $\mathrm{n}$ & 1.31 & $\mathrm{n}$ & 1.31 & \\
\hline $\mathrm{P}_{1}(1.2 \mathrm{~cm})$ & 1.95 & g-j & 1.54 & $\mathrm{~m}$ & 1.75 & G-I & 1.62 & $\mathrm{~m}$ & 1.88 & $g-j$ & 1.75 & F-G \\
\hline $\mathrm{P}_{2}(1.8 \mathrm{~cm})$ & 1.90 & i-k & 1.65 & $\mathrm{~m}$ & 1.78 & I & 1.67 & $1-m$ & 1.90 & $g-i$ & 1.79 & F-G \\
\hline $\mathrm{P}_{3}(2.4 \mathrm{~cm})$ & 1.98 & $e-h$ & 1.67 & $1-m$ & 1.83 & E-G & 1.80 & i-1 & 1.97 & $e-h$ & 1.89 & C-D \\
\hline Algae & 1.83 & i-k & 1.67 & $1-m$ & 1.75 & H-I & 1.73 & $\mathrm{k}-\mathrm{m}$ & 1.63 & i-1 & 1.68 & $\mathrm{G}$ \\
\hline $\mathrm{P}_{1}+$ Algae & 1.93 & $g-i$ & 1.82 & $\mathrm{i}-\mathrm{k}$ & 1.88 & D-f & 1.63 & $\mathrm{~m}$ & 1.83 & h-k & 1.73 & G \\
\hline $\mathrm{P}_{2}+$ Algae & 1.99 & $e-g$ & 1.89 & $g-j$ & 1.94 & C-D & 1.73 & $\mathrm{k}-\mathrm{m}$ & 1.95 & $f-h$ & 1.84 & E-F \\
\hline $\mathrm{P}_{3}+$ Algae & 2.00 & e-g & 1.96 & $f-h$ & 1.98 & $\mathrm{C}$ & 1.87 & g-k & 2.10 & c-e & 1.99 & $\mathrm{C}$ \\
\hline Yeast & 1.88 & $h-j$ & 1.69 & $g-j$ & 1.79 & G-I & 1.75 & $\mathrm{j}-\mathrm{m}$ & 1.94 & f-h & 1.85 & E-F \\
\hline$P_{1}+$ Yeast & 1.83 & i-k & 1.78 & $\mathrm{j}-1$ & 1.81 & G-I & 1.78 & i-1 & 2.05 & $d-f$ & 1.92 & C-E \\
\hline $\mathrm{P}_{2}+$ Yeast & 2.11 & $b-d$ & 1.83 & $\mathrm{i}-\mathrm{k}$ & 1.97 & D-F & 1.80 & i-1 & 2.12 & $\mathrm{p}-\mathrm{d}$ & 1.96 & C-D \\
\hline $\mathrm{P}_{3}+$ Yeast & 2.15 & $b-c$ & 2.00 & d-g & 2.08 & B & 1.87 & g-k & 2.13 & $b-d$ & 2.00 & $\mathrm{C}$ \\
\hline Algae + Yeast & 1.92 & $g-i$ & 1.87 & $h-j$ & 1.90 & $\mathrm{C}-\mathrm{F}$ & 1.78 & i-1 & 2.00 & d-g & 1.89 & C-D \\
\hline $\mathrm{P}_{1}+$ Algae + Yeast & 2.08 & c-e & 1.84 & $\mathrm{i}-\mathrm{k}$ & 1.96 & C-D & 2.22 & $a-c$ & 2.23 & $\mathrm{a}-\mathrm{c}$ & 2.23 & B \\
\hline $\mathrm{P}_{2}+$ Algae + Yeast & 2.20 & $a-b$ & 2.07 & $c-f$ & 2.14 & B & 2.22 & $\mathrm{a}-\mathrm{c}$ & 2.25 & $a-b$ & 2.24 & B \\
\hline P3 + Algae + Yeast & 2.28 & $\mathrm{a}$ & 2.18 & $\mathrm{a}-\mathrm{c}$ & 2.23 & A & 2.35 & $\mathrm{a}$ & 2.32 & $\mathrm{a}$ & 2.34 & A \\
\hline Means & 1.94 & A & 1.78 & B & & & 1.80 & B & 1.96 & & & \\
\hline
\end{tabular}

Leaf phosphorus $(P)$ content

Under this investigate, phosphorus contents was insignificant differed between the two citrus rootstocks in season 2011. While, Table 6 showed that

Egypt. J. Hort. Vol. 41, No. 2 (2014) 
leaf $(\mathrm{P})$ content of Sour orange seedlings had the highest percentage in season 2012. Seedlings under control treatment appeared to gain the lowest significant content of $(\mathrm{P})$ during both seasons. Phosphorus content per leaf was significantly affected by increasing P-rate. Application of yeast or algae alone improved the percentage of $(\mathrm{P})$ in leaf and this increment was more enhanced by $(\mathrm{P})$ addition. Accordingly, leaf phosphorus content (\%) had significantly the outmost by using the highest dose of $(\mathrm{P})$ plus algae and yeast on the two seasons for the two rootstocks.

TABLE 6. Effect of some stimulants on leaf phosphorus content of two citrus rootstocks during 2011 and 2012 seasons.

\begin{tabular}{|c|c|c|c|c|c|c|c|c|c|c|c|c|}
\hline \multirow{4}{*}{$\begin{array}{l}\begin{array}{c}\text { Stimulative } \\
\text { substances }\end{array} \\
\text { Control }\end{array}$} & \multicolumn{12}{|c|}{ Phosphorus (\%) } \\
\hline & \multicolumn{6}{|c|}{ 2011season } & \multicolumn{6}{|c|}{2012 season } \\
\hline & \multicolumn{2}{|c|}{$\begin{array}{c}\text { Sour } \\
\text { orange }\end{array}$} & \multicolumn{2}{|c|}{$\begin{array}{l}\text { Volkamer } \\
\text { lemon }\end{array}$} & \multicolumn{2}{|c|}{ Means } & \multicolumn{2}{|c|}{ Sour orange } & \multicolumn{2}{|c|}{$\begin{array}{c}\text { Volkamer } \\
\text { lemon }\end{array}$} & \multicolumn{2}{|c|}{ Means } \\
\hline & 0.14 & n-o & 0.11 & $\mathrm{o}$ & 0.13 & $\mathrm{~J}$ & 0.12 & $\mathrm{~m}$ & 0.09 & $\mathrm{~m}$ & 0.11 & \\
\hline$P_{1}(1.2 \mathrm{~cm})$ & 0.21 & $\mathrm{~g}-\mathrm{k}$ & 0.17 & $m-n$ & 0.19 & G-I & 0.19 & ij & 0.15 & 1 & 0.17 & G \\
\hline $\mathrm{P}_{2}(1.8 \mathrm{~cm})$ & 0.22 & $\mathrm{f}-\mathrm{k}$ & 0.21 & $\mathrm{~g}-\mathrm{k}$ & 0.22 & F-G & 0.21 & $f-i$ & 0.19 & $\mathrm{ij}$ & 0.20 & $\mathrm{EF}$ \\
\hline $\mathrm{P}_{3}(2.4 \mathrm{~cm})$ & 0.24 & d-g & 0.22 & $f-j$ & 0.23 & $\mathrm{CD}$ & 0.23 & $e-g$ & 0.24 & $e-g$ & 0.24 & CD \\
\hline Algae & 0.18 & $1-\mathrm{m}$ & 0.16 & m-n & 0.17 & I & 0.15 & $\mathrm{kl}$ & 0.17 & $\mathrm{j}-1$ & 0.16 & G \\
\hline $\mathrm{P}_{1}+$ Algae & 0.19 & $\mathrm{k}-\mathrm{m}$ & 0.21 & $\mathrm{~g}-\mathrm{k}$ & 0.20 & F-H & 0.19 & $\mathrm{ij}$ & 0.20 & $g-i$ & 0.20 & $\mathrm{EF}$ \\
\hline $\mathrm{P}_{2}+$ Algae & 0.21 & $\mathrm{j}-\mathrm{m}$ & 0.22 & $f-j$ & 0.22 & D-F & 0.23 & $\mathrm{j}-1$ & 0.23 & $e-g$ & 0.23 & $\mathrm{CD}$ \\
\hline $\mathrm{P}_{3}+$ Algae & 0.22 & $f-j$ & 0.24 & d-h & 0.23 & $\mathrm{DE}$ & 0.24 & $e-g$ & 0.23 & $\mathrm{e}-\mathrm{g}$ & 0.24 & CD \\
\hline Yeast & 0.19 & $\mathrm{i}-\mathrm{m}$ & 0.17 & $\mathrm{~m}$ & 0.18 & HI & 0.17 & $\mathrm{j}-1$ & 0.19 & $\mathrm{i}-\mathrm{k}$ & 0.18 & FG \\
\hline$P_{1}+$ Yeast & 0.23 & e-h & 0.24 & d-h & 0.24 & $\mathrm{CE}$ & 0.23 & $e-g$ & 0.21 & $f-i$ & 0.22 & $\mathrm{DE}$ \\
\hline $\mathrm{P}_{2}+$ Yeast & 0.24 & $i-m$ & 0.24 & d-g & 0.24 & $\mathrm{CD}$ & 0.24 & $d-f$ & 0.22 & $\mathrm{ij}$ & 0.23 & $\mathrm{CD}$ \\
\hline $\mathrm{P}_{3}+$ Yeast & 0.26 & d-e & 0.25 & $c-f$ & 0.26 & $\mathrm{BC}$ & 0.26 & de & 0.24 & $d-f$ & 0.25 & $\mathrm{C}$ \\
\hline Algae + Yeast & 0.20 & b-e & 0.21 & $\mathrm{~g}-\mathrm{k}$ & 0.21 & F-H & 0.20 & $h-j$ & 0.19 & $\mathrm{ij}$ & 0.20 & $\mathrm{~F}$ \\
\hline $\mathrm{P}_{1}+$ Algae + Yeast & 0.27 & $a-d$ & 0.28 & $\mathrm{a}-\mathrm{c}$ & 0.28 & $\mathrm{AB}$ & 0.29 & $a-c$ & 0.26 & c-e & 0.28 & B \\
\hline $\mathrm{P}_{2}+$ Algae + Yeast & 0.28 & $\mathrm{a}-\mathrm{c}$ & 0.29 & $a b$ & 0.29 & A & 0.30 & $a b$ & 0.27 & $b-d$ & 0.29 & $\mathrm{AB}$ \\
\hline $\mathrm{P}_{3}+$ Algae + Yeast & 0.29 & $a b$ & 0.30 & $\mathrm{a}$ & 0.30 & A & 0.31 & $\mathrm{a}$ & 0.29 & $a b$ & 0.30 & \\
\hline Means & 0.22 & n.s & 0.22 & $\mathrm{n} . \mathrm{s}$ & & & 0.22 & A & 0.21 & B & & \\
\hline
\end{tabular}

Leaf potassium (K) content

Potassium contents were significantly affected by citrus rootstocks in the two seasons but with no stable trend. Since, Volkamer or Sour orange interchanged recording the higher leaf $k$ content in the two seasons of study (Table 7). In addition, the highest dose of $(\mathrm{P})$ plus algae and yeast was significantly resulted in the uppermost $\mathrm{K}$ content. It also increased linearly as $(\mathrm{P})$ doses rose during both seasons. Yeast had more positive effect on leaf nitrogen content compared to algae application. The combined treatments increased significantly $(\mathrm{K})$ leaf content compared to the single ones. On the other hand, the lowest leaf content recorded with both rootstocks was in control treatment. 
TABLE 7. Effect of some stimulants on leaf Potassium content of two citrus rootstocks during 2011 and 2012 seasons.

\begin{tabular}{|c|c|c|c|c|c|c|c|c|c|c|c|c|}
\hline \multirow{4}{*}{$\begin{array}{l}\text { Stimulative } \\
\text { Substances } \\
\text { Control }\end{array}$} & \multicolumn{12}{|c|}{ Potassium (\%) } \\
\hline & \multicolumn{6}{|c|}{ 2011season } & \multicolumn{6}{|c|}{2012 season } \\
\hline & \multicolumn{2}{|c|}{$\begin{array}{c}\text { Sour } \\
\text { orange }\end{array}$} & \multicolumn{2}{|c|}{$\begin{array}{c}\begin{array}{l}\text { Volkamer } \\
\text { lemon }\end{array} \\
\end{array}$} & \multicolumn{2}{|c|}{ Means } & \multicolumn{2}{|c|}{$\begin{array}{c}\text { Sour } \\
\text { orange }\end{array}$} & \multicolumn{2}{|c|}{$\begin{array}{c}\begin{array}{l}\text { Volkamer } \\
\text { lemon }\end{array} \\
\end{array}$} & \multicolumn{2}{|c|}{ Means } \\
\hline & 1.07 & $\mathrm{n}$ & 1.09 & $\mathrm{n}$ & 1.08 & $\mathrm{~K}$ & 1.10 & $q$ & 1.02 & $\mathrm{r}$ & 1.06 & I \\
\hline $\mathrm{P}_{1}(1.2 \mathrm{~cm})$ & 1.25 & $\mathrm{~m}$ & 1.34 & $\operatorname{lm}$ & 1.30 & $\mathrm{~J}$ & 1.39 & op & 1.34 & $\mathrm{p}$ & 1.37 & $\mathrm{H}$ \\
\hline $\mathrm{P}_{2}(1.8 \mathrm{~cm})$ & 1.30 & $\operatorname{lm}$ & 1.54 & $\mathrm{ij}$ & 1.42 & I & 1.50 & $\operatorname{lm}$ & 1.53 & K 1 & 1.51 & $\mathrm{~F}$ \\
\hline $\mathrm{P}_{3}(2.4 \mathrm{~cm})$ & 1.52 & $\mathrm{ij}$ & 1.67 & $\mathrm{gh}$ & 1.59 & $\mathrm{~F}$ & 1.65 & g-i & 1.64 & hi & 1.65 & $\mathrm{E}$ \\
\hline Algae & 1.29 & $\operatorname{lm}$ & 1.38 & $\mathrm{k} 1$ & 1.33 & $\mathrm{~J}$ & 1.41 & no & 1.39 & op & 1.40 & $\mathrm{GH}$ \\
\hline $\mathrm{P}_{1}+$ Algae & 1.53 & fg & 1.44 & $\mathrm{jk}$ & 1.49 & $\mathrm{HI}$ & 1.57 & $\mathrm{jk}$ & 1.46 & $\mathrm{mn}$ & 1.52 & $\mathrm{~F}$ \\
\hline $\mathrm{P}_{2}+$ Algae & 1.70 & fg & 1.66 & gh & 1.68 & DE & 1.65 & gh & 1.60 & ij & 1.63 & $\mathrm{E}$ \\
\hline $\mathrm{P}_{3}+$ Algae & 1.83 & $\mathrm{~cd}$ & 1.80 & $d-f$ & 1.82 & $\mathrm{C}$ & 1.71 & $d-f$ & 1.69 & e-g & 1.70 & $\mathrm{D}$ \\
\hline Yeast & 1.50 & ij & 1.51 & $\mathrm{jj}$ & 1.51 & $\mathrm{GH}$ & 1.42 & no & 1.45 & $\mathrm{mn}$ & 1.44 & G \\
\hline $\mathrm{P}_{1}+$ Yeast & 1.64 & gh & 1.51 & $\mathrm{ij}$ & 1.58 & FG & 1.67 & $f-h$ & 1.56 & $\mathrm{jk}$ & 1.62 & E \\
\hline $\mathrm{P}_{2}+$ Yeast & 1.72 & $e-g$ & 1.72 & $e-g$ & 1.72 & $\mathrm{D}$ & 1.75 & $\mathrm{~cd}$ & 1.68 & $f-h$ & 1.72 & $\mathrm{D}$ \\
\hline $\mathrm{P}_{3}+$ Yeast & 1.81 & de & 1.80 & $d-f$ & 1.81 & $\mathrm{C}$ & 1.78 & $\mathrm{c}$ & 1.74 & c-e & 1.76 & $\mathrm{C}$ \\
\hline Algae + Yeast & 1.66 & $\mathrm{gh}$ & 1.59 & hi & 1.63 & $\mathrm{EF}$ & 1.53 & $\mathrm{kl}$ & 1.54 & $\mathrm{kl}$ & 1.54 & $\mathrm{~F}$ \\
\hline $\mathrm{P}_{1}+$ Algae $+\underline{\text { Yeast }}$ & 1.81 & de & 1.93 & $\mathrm{bc}$ & 1.87 & $\mathrm{BC}$ & 1.83 & $\mathrm{~b}$ & 1.75 & $\mathrm{~cd}$ & 1.79 & $\mathrm{C}$ \\
\hline $\mathrm{P}_{2}+$ Algae + Yeast & 1.86 & $\mathrm{~cd}$ & 1.99 & $a b$ & 1.93 & $\mathrm{AB}$ & 1.88 & $\mathrm{~b}$ & 1.87 & $\mathrm{~b}$ & 1.88 & B \\
\hline $\mathrm{P}_{3}+$ Algae + Yeast & 1.92 & $\mathrm{bc}$ & 2.05 & $\mathrm{a}$ & 1.99 & A & 1.95 & $\mathrm{a}$ & 1.96 & $\mathrm{a}$ & 1.96 & A \\
\hline Means & 1.58 & B & 1.62 & A & & & 1.60 & A & 1.57 & B & & \\
\hline
\end{tabular}

\section{Discussion}

Generally, the growth stimulators tested in this research had a positive enhancing effect varied in their impact. Where, phosphorus was the superlative one as it achieved the uppermost outcomes of Sour orange and Volkamer lemon rootstock seedling parameters. Where, most of the considered parameters increased linearly by increasing $(\mathrm{P})$ doses in both rootstocks (Al-Karaki, 2011 and Abdelaal et al., 2013). Consequently, (P) fertilizer instigated a significant increase of growth measurements (Boughalleb et al., 2011 and Zambrosi et al., 2011). Furthermore, the general trends of unaccompanied algae and/or yeast on any of rootstock seedlings parameters comparing to the $(\mathrm{P})$ combined ones could signify to the principle role of phosphorus in such aspects. These previous advantages may be due to the function of phosphorus in plants as it is vital to every living plant cell. It is involved in several key plant functions, including energy transfer, photosynthesis, transformation of sugars and starches, nutrient Egypt. J. Hort. Vol. 41, No. 2 (2014) 
movement within the plant and transfer of genetic characteristics from one generation to the next (Better, 1999). However, yeast extract increased the vegetative parameters resulted in roots dry weight (Hamza, 2013). Whereas, the positive action of active dry yeast on leaf area and its contents of various nutrients could be attributed to its content of cytokinins and vitamin B as well as its important role in building up carbohydrates. These previous benefits of such materials surely were reflected on enhancing both cell division and cell enlargement (Abd El-Moniem et al., 2008).

Despite algae attained the least positive effect among the examined materials, yet it improved vegetative growth plant height, stem thickness, leaves number per plant and plant dry weight (Ismail et al., 2011). Whereas, the various positive effects of applying algae extract may attributable to its contains of $\mathrm{N}, \mathrm{P}, \mathrm{K}, \mathrm{Ca}$, $\mathrm{Mg}, \mathrm{S}, \mathrm{Zn}, \mathrm{Fe}, \mathrm{Mn}, \mathrm{Cu}, \mathrm{Mo}$, Co, some growth regulators, polyamines and vitamins. So, it is applied to improve nutritional status and vegetative growth (Abd El-Motty et al., 2010). Subsequently, the studied parameters were enhanced with combination of the three stimulants applications followed by bilateral combination then application with either substance alone.

It was cleared from the earlier data that, enhanced leaf content of N, P and K, which enriched by apply stimulants (phosphorus, yeast and algae), intensified growth parameters such as stem length, stem thickness, leaf area and dry weight of root. These positive effects in growth were due to the role of this element in plant functioning (Abbas and Fares, 2008). Nitrogen has many functions in plant life. Being a part of proteins, $\mathrm{N}$ is an important constituent of protoplasm. It is responsible for the biosynthesis of enzymes, nucleoproteins, amino acid, amines, amino sugars, polypeptides, chlorophylls and encourages cell divisions (Nijjar, 1985). While, phosphorus is necessary for many life processes such as photosynthesis, synthesis and breakdown of carbohydrates and the transfer of energy within the plant (Obreza, 2001). Further, potassium is necessary for basic physiological functions such as formation of sugars and starch, synthesis of proteins and cell division and growth (Abbas and Fares, 2008). This is reflected on growth parameters. Consequently, increased $(\mathrm{P})$ fertilizer rate resulted in improving leaf N, P and K content (Barakat, et al., 2012 and Mohamed et al., 2013). Enhancement leaf N, P and $\mathrm{K}$ content with application is attributed to yeast or algae extract as they contain many different nutrients (Mostafa and ElHosseiny, 2001), by increasing soil organism's activity and so increasing availability of nutrient elements and consequence their uptake (Mustafa and ElShazly, 2013) and indirectly by improving root growth (Hafez et al., 2013) and these enhancing in root absorption. This was correspondingly confirmed by Abdelaal et al. (2013) and Ismail et al. (2011) where they noted that, applying yeast and algae was very effective in improving leaf $\mathrm{N}, \mathrm{P}$ and $\mathrm{K}$ content.

However, the interaction between Sour orange and Volkamer lemon rootstock seedlings had significant performance trends. Where, in spite of the vigor habit of Volkamer lemon rootstock which appeared in its stem seedlings 
height, Sour orange seedlings utmost them in average \& total leaf and root dry weight. The high-handed for the Sour orange in the later parameter may clarify upon the advantage basis; the utilized moist media in this investigation was the favorable media for such rootstock (Hemeda, 2014) which reflected in augmenting the vegetative features.

To conclude, growth stimulants significantly affected plant dry weight accumulation and partitioning into stems, leaves, and roots, which may have an impact on transplants growth consequently shorten the time they need to be fit for grafting. The superlative and the indispensable one was the phosphorus followed by yeast with important rule in enhancing seedlings growth, while the diatoms (algae) has a positive affect less significantly than the other two stimulants. So, it could be recommended that utilizing $(\mathrm{P})$ is vital for nursery nutrition, yeast has a considerable induce in this concern and the best results under this investigation-were obtained by applying highest dose of $(\mathrm{P})$ plus algae and yeast.

\section{References}

Abbas, F. and Fares, A. (2008) Best management practices in citrus production. Tree For. Sci. Biotech, 3, 1-11.

Abd El-Moniem, E.A. and Abd-Allah, A.S.E. (2008) Effect of green alga cells extract as foliar spray on vegetative growth, yield and berries quality of Superior grapevines. Amer. Eurasian J. Agric. \& Environ. Sci., 4 (4), 427-433.

Abd El-Motty, E.Z., Shahin, M. F. M., El-Sheikh, M. H. and Abd El-Migeed, M. M. M. (2010) Effect of algae extract and yeast application on growth, nutritional status, yield and fruit quality of Keitte mango trees. Agriculture and biology Journal of North America, 1(3), 421-429.

Abdelaal, A.H.M., Ahmed, F.F., El-Masry, E.M.A. and Abdallah, A.A. (2013) Using Potassium Sulphur as well as organic and biofertilization for alleviating the adverse effects of salinity on growth and fruiting of Valencia orange trees. Stem Cell, 4(4), 27-32.

Agriculture Census of 2012 Ministry of Agriculture and land Reclamation., Egypt. Published, June (2013).

Al-Karaki, G.N. (2011) The effect of Arbuscular Mycorrhizal fungi on the establishment of Sour orange (Citrus aurantium) under different levels of phosphorus mineral nutrition of fruit crops, 984,103-108.

Barakat, M.R., Yehiaand, T.A., Sayed, B.M. (2012) Response of Newhall naval orange to bio-organic fertilization under newly reclaimed area conditions I: Vegetative growth and nutritional status. J. Horti. Sci.\& Ornamental Plants, 4 (1), 18-25.

Better (1999) Functions of phosphorus in plants. Crops, 83(1), 6- 7. 
Bleasdale, L.K.A. (1987) Plant physiology in relation to horticulture. ELBS edition. The Macmillan press LTD. Associated companies in Delhi, Hong Kong Lagos and Singapore.

Boughalleb, F., Mhamdi, M. and Hajlaoui, H. (2011) Response of young citrus trees to NPK fertilization under greenhouse and field conditions. Agric. J., 6(3), 66-73.

El-Kassas, S.E. (1984) Effect of iron nutrition on the growth, yield, fruit quality and leaf composition of Balady lime trees grown on sandy calcareous soils. J. Plant Nutrition, 7, 301-311.

Hafez, A.M., Saleh, M. A. and El-Lethy, S. R. (2013) Response of some seedlings olive cultivars to foliar spray of yeast and garlic extracts with or without vascular arbuscular mycorrhizal fungi. World Appl. Sci. J., 24 (9), 1119-1129.

Hamza, M.A. (2013) Effect of yeast extract (biotic elicitor) and incubation periods on selection of Lupinustermis explants in vitro. J. Appl. Sci. Res., 9(7), 4186-4192.

Hegab, M.Y., Sharawy, A. M. A. and El- Saida, S. A. G. (2005) Effect of algae extract and mono potassium phosphate on growth and fruiting of Balady orange trees. BullFac. Agric. Cairo Univ., 56(1), 107-120.

Hemeda, S.H. (2014) Effect of rootstock and soil type on tree growth and fruit quality of some orange varieties. M.Sc. Thesis, Fac. Agric, Cairo Univ., Egypt.

Ibrahim, D.S.M. (2007) Alleviation of salinity hazards in Valencia orange plants budded on two rootstocks by NPK nutrient amendments. M.Sc. Thesis, Fac. Agric., Alexandria Univ., Egypt.

Ismail, O.M., Dakhly, O.F. and Ismail, M.N. (2011) Influence of some bacteria strains and algae as biofertilizers on growth of bitter orange seedlings. Australian J. Basic \& Appl. Sci., 5(11), 1285-1289.

Jackson, N.L. (1958) "Soil Chemical Analysis", Constable. Ltd. Co. London p.498.

Labanauskas, C.K. (1966) Effect of orange leaf washing techniques on removal of surface contaminants and nutrient losses. Proc. Amer. Soc. Hort. Sci., 89, 201-210.

Latif, F.G. (2004) Citrus improvement program-citrus nurseries forecast-discussion. Citrus Res. division-HRI, ARC, MALR Paper \# 11.

Mohamed, H.M., Al-Kamar, F.A. and Abd-Elall, A.M. (2013) Effect of magnetite and some biofertilizer application on growth and yield of Valencia orange trees under El Bustan condition. Nature and Sci., 11(6), 46-61.

Mostafa, M.F. and El-Hosseiny, A.A. (2001) Influence of spraying active dry yeast solution on growth, yield, fruit quality and leaf NPK content of Washington navel orange trees. J. Agric. Sci. Mansoura Univ., 26(10), 6293-6305.

Mustafa, N.S. and El-Shazly, S.M. (2013) Impact of some biostimulant substances on growth parameters of Washington navel orange trees. Middle East J. Appl. Sci., 3(4), 156-160.

Egypt. J. Hort. Vol. 41, No. 2 (2014) 
Nagodawithana, W.T. (1991) "Yeast Technology", Universal Foods Corporation. Milwaukee, Wisconsin, Published by Van Nostrand Reinhold, New York, p.273.

Nijjar, G.S. (1985) Nutrition of Fruit Trees. Kalayani publishers. New Delhi Ludhiana, pp.10- 52, 173- 270.

Obreza, T.A. (2001) Effects of $\mathrm{P}$ and $\mathrm{K}$ fertilization on young citrus tree growth. Cooperative extension service, Univ. of Florida, inst. of food and agric. sci., Florida, pp. 1-3.

Piper, C.S. (1950) "Soil and Plant Analysis". Inter. Sci, palb, New York, p. 368.

Raghothama, K.G. and Karthikeyan, A.S. (2005) Phosphate acquisition. In Root Physiology: from Gene to Function. Springer Netherlands, 37-49.

Snedecor, G.W. and Cochran, W.G. (1989) "Statistical Methods", $8^{\text {th }}$ ed., Ames. Iowa State Univ. Press, Iowa, U.S.A.

Vanshouwenburg, J.C.H. and Walinga, I. (1978) "Methods of Analysis for Plant Material", Agric. Univ., Wagenjnen, Netherlands.

Waller, C. and Duncan, D.B. (1969) Multiple ranges and multiple tests. Biometrics, 11, $1-24$.

Water \& Environment Research Institute. Analysis \& studies component (2010) Agricultural Research Center, Ministry of Agriculture and land Reclamation, Egypt.

Zambrosi, F.C.B., Mattos, D.J. and Syvertsen, J.P. (2011) Plant growth, leaf photosynthesis, and nutrient-use efficiency of citrus rootstocks decrease with phosphate supply. J. Plant Nutr. Soil Sci., 174, 487-495.

(Received 4 /9 /2014; accepted 19/10/2014) 
إستجابة شتلات بعض أصول الموالح لإضافة مواد منشطة للنمو

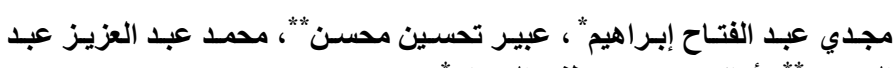

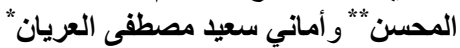

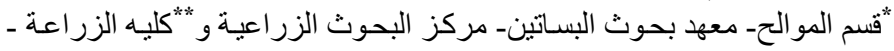
جامعه القاهرة ـ القاهرة - مصر بحر.

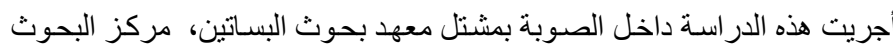

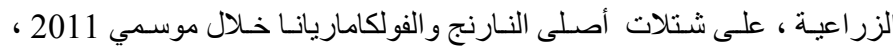

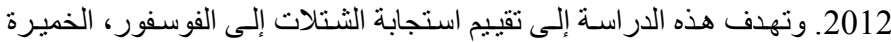

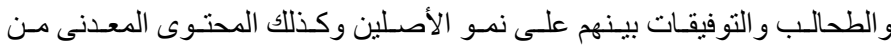
عناصر "النيتروجين، الفوسفور و البوتاسيوم

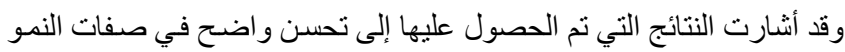

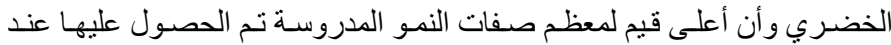

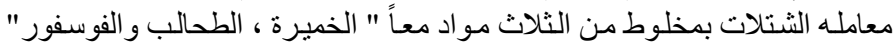

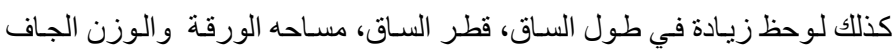

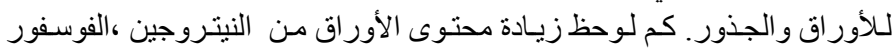
و البوتاسيوم زيادة معنوية عند إضافه هذا المخلوط خلادل موسمى الدر اسة.

وبـللك يمكن التوصية باسـتخدام الخلبط مـن الخميرة ، الطحالب و الفوسـفور

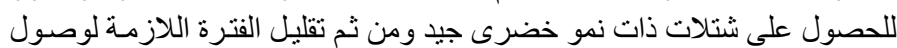

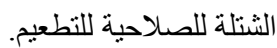

الكلمات الداله: أصول الموالح، النارنج، الفولكاماريانا، النمو الخضرى، الفوسفور، خميرة جافه نشطه وطحالب. اله البول 